\title{
UNA PROPUESTA DE PROTECCIÓN PARA UN PATRIMONIO MUY FRÁGIL: LOS SITIOS CON ARTE RUPESTRE DE ANDALUCÍA
}

\section{A PROPOSAL OF PROTECTION FOR A VERY FRAGILE HERITAGE: ROCK ART SITES IN ANDALUSIA}

\author{
por \\ ISABEL SANTANA FALCÓN Y \\ SANDRA RODRÍGUEZ DE GUZMÁN SÁNCHEZ
}
RESUMEN Se explican los criterios de encargo y los resultados del trabajo realizado para la Dirección General de Bienes Culturales de la Consejería de Cultura de la Junta de Andalucía en orden a determinar posibles riesgos de deterioro en las estaciones con arte rupestre del municipio de Jaén.

\begin{abstract}
The character of the assignment is explained, followed by the results of the work undertaken by the Dirección General de Bienes Culturales of the Consejería de Cultura of the Junta de Andalucía, with the aim of gauging the possible risks of degradation to rock art sites in the Término Municipal of Jaén.
\end{abstract}

Palabras claves Arte rupestre, Patrimonio Arqueológico, Jaén, Protección, Conservación.

Key words $\quad$ Risks of degradation, rock art sites, Jaén.

Tratar la protección de la totalidad del Patrimonio Arqueológico desde la perspectiva de la Administración implica la necesidad de elaborar criterios para el desarrollo de una programación.

Las acciones que se derivan de los conceptos de protección-conservación, unas veces responden al desarrollo de la legislación vigente, no sólo en materia de Patrimonio Histórico sino en cualquier tipo de materia que regule el uso y transformación de suelo donde se encuentra el Patrimonio Arqueológico, ${ }^{1}$ mediante

\footnotetext{
1. Sin citar su desarrollo reglamentario, la legislación vigente relativa a este tema es la siguiente: —Legislación de Patrimonio Histórico: Ley 16/1985 del Patrimonio Histórico Español, Ley 1/91 del Patrimonio Histórico de Andalucía.
} 
la aplicación de sus figuras de protección (declaraciones de BIC, inscripciones en el Catálogo General del Patrimonio Histórico de Andalucía) o del desarrollo de sus preceptos relativos a la conservación (Planes Especiales de Protección, Catálogos, Evaluaciones de Impacto Ambiental etc). Otras veces las acciones responden a la ejecución de proyectos para aplicar medidas concretas promovidas por diferentes administraciones: estudios de diagnóstico, cerramientos y vallados, señalizaciones etc.

Si bien todas éstas acciones sería conveniente ejecutarlas en todos los elementos pertenecientes al Patrimonio Arqueológico, la ingente cantidad de dichos bienes (aproximadamente 12.500 en nuestra Comunidad Autónoma, según la base de datos ARQUEOS integrada en el SIPHA ${ }^{2}$ obliga a la Administración a elaborar programaciones que ordenen no sólo la elección de los bienes sobre los que hay que actuar y aplicar los recursos de manera más inmediata, sino también qué tipo de acción es la más necesaria para su correcta Tutela.

De todos los bienes pertenecientes al Patrimonio Arqueológico, el caso que tratamos, el arte rupestre, es un caso singular. Es singular en cuanto a su situación legal, ya que tiene asignada, por ley, la máxima categoría de protección en el artículo 40.2 de la Ley 16/85 del Patrimonio Histórico Español. ${ }^{3}$ Asimismo, se incluyó en la "Lista de Patrimonio Mundial" el Arte Rupestre del Arco Mediterráneo de la Península Ibérica (Kyoto, diciembre de 1998).

Es especial también, por su ubicación ya que, de ningún modo, podemos aislar el medio natural dónde se encuentran de la actividad humana que originó tales manifestaciones, generándose así el concepto patrimonial de entorno de protección, en unos casos, o de área de impluvio, en otros (Fortea Pérez ed. 1993). Esa misma relación entre el medio natural -su soporte- y las pinturas las dota de extrema fragilidad, y se hace necesario diseñar tratamientos de conservación específicos para ellas.

En ese sentido, de relación medio físico-patrimonio arqueológico, son de suma importancia las actuaciones preventivas que en materia de protección se puedan desarrollar ya que constituyen la clave para promover acciones ordenadas, programadas y sin las presiones que ciertos usos y transformaciones del suelo, futuros o consolidados, puedan ejercer sobre ellas y que, en algunos casos, pueden llevar a deterioros irreversibles. El tratamiento bibliográfico que hasta ahora se ha dado al arte rupestre excepcionalmente ha tratado aspectos relativos a su protección, pero en ningún caso criterios para la delimitación de ámbitos objeto de tutela, salvo excepciones (Fortea Pérez ed. 1993). Por tanto se carecía de experiencias concretas que permitiesen ordenar las actuaciones tutelares de la administración en un vasto territorio como el nuestro.

"En Andalucía se han documentado hasta ahora 467 estaciones con manifestaciones artísticas parietales, según recoge el Catálogo de Yacimientos con Pinturas Rupestres. ${ }^{4}$ Su distribución provincial es la siguiente: 64 se encuentran en 10 municipios de la provincia de Almería; 12 municipios de la provincia de Cádiz albergan 129 yacimientos; Córdoba cuenta con 14 estaciones con arte rupestre distribuidas en 7 Términos Municipales; 38 yacimientos se han descubierto en 12 términos municipales de Granada; Jaén alberga

— Legislación del Suelo: RDL 1/1992 Texto Refundido sobre Régimen de Suelo y Ordenación Urbana, Ley 1/97 por la que se adoptan con carácter urgente y transitorio disposiciones en materia de Régimen de Suelo y Ordenación Urbana.

— Legislación del Territorio: Ley 1/1994, de Ordenación del Territorio de la Comunidad Autónoma Andaluza.

— Legislación de Medio Ambiente: Ley 4/1989 de Espacios Naturales Protegidos y Flora y Fauna Silvestre; Ley 2/1989 por la que se aprueba el Inventario de Espacios Naturales Protegidos y se establecen medidas adicionales para su protección; RDL 1302/1986 de Evaluación de Impacto Ambiental y sus modificaciones, contenidas en el RDL 9/2000 y Ley 6/2001; Ley 7/1994, de Protección Ambiental.

2. Sistema de Información del Patrimonio Histórico de Andalucía (SIPHA). Consejería de Cultura de la Junta de Andalucía.

3. Quedan declarados bienes de interés cultural por ministerio de esta ley las cuevas, abrigos y lugares que contengan manifestaciones de arte rupestre.

4. Catálogo de Yacimientos con Pinturas Rupestres. 1994. Elaborado por M. Más Cornellá por encargo de la Dirección General de Bienes Culturales. 
el mayor número de abrigos con pinturas rupestres prehistóricas, 165, repartidas en 16 municipios; finalmente, 20 Términos Municipales albergan 59 yacimientos en Málaga.

Tal y como se indica en la introducción del catálogo citado, su información se basa principalmente en la recopilación bibliográfica, ampliada con documentación complementaria de la propia Consejería de Cultura. Dicha recopilación se efectuó de forma objetiva y sin valorar los datos que aportaban las diferentes fuentes consultadas; la información se ordenó en el modelo de fichas para yacimientos arqueológicos con que contaba la Consejería en ese momento. Igualmente, la localización debía considerarse provisional, porque la escala utilizada -1:50.000- no es la más indicada para emplazar con precisión yacimientos de estas características. En definitiva, podemos considerar este documento como el resultado de un primer acercamiento a la realidad del arte rupestre de Andalucía.

Como consecuencia del carácter preliminar de este trabajo, los contenidos en relación a los distintos ámbitos de la Tutela eran escasos. La información que ofrece para la Investigación representa un punto de partida correcto, antecedente de un desarrollo más profundo y extenso de otras labores de conocimiento, básicas para la aplicación y ejercicio de otros campos de la gestión de los bienes culturales. Desde la óptica de la Conservación, los datos que aporta son escasos, casi siempre breves síntesis de las noticias que ofrece la bibliografía científica. Respecto a la Protección de estos yacimientos, las únicas noticias que se incluyen son las relacionadas con la localización de algunos de ellos en Espacios Naturales, así como su ubicación en áreas protegidas en los distintos planes especiales de protección del medio físico. Ningún otro aspecto ligado a la protección, tales como cuestiones relacionadas con el planeamiento urbanístico municipal, la propiedad del suelo, la determinación del territorio afectado por el yacimiento y su entorno, etc. se había trabajado. Por último, no se hacía ninguna referencia a la posibilidad, o no, de emprender algún tipo de actuación destinada a la Difusión de estos bienes patrimoniales, o de modificar actividades de estas características que se estuvieran llevando a cabo" (Rodríguez de Guzmán Sánchez et al. 2002b).

De este modo, se hacía evidente la necesidad de profundizar y ampliar nuestro conocimiento de las estaciones con arte rupestre de Andalucía a todos los ámbitos de la Tutela, y prioritariamente a los aspectos preventivos de la Protección y la Conservación. En este sentido, la publicación en 1992 de La Carta de Riesgo (ISTITUTO CENTRALE PER IL RESTAURO, 1992) supuso un paso adelante, porque conocer los instrumentos que en otros lugares se están utilizando, y analizar su estructura, permitió avanzar en su adaptación a las necesidades específicas de nuestro patrimonio (Rodríguez de Guzmán Sánchez et al. 2002a).

Así pues, y con el objetivo de buscar herramientas eficaces para alcanzar los objetivos programados desde el ámbito concreto de la Protección, se propusieron las siguientes líneas de actuación:

1. A nivel general, y como cuestión esencial para el desarrollo de las siguientes propuestas, existía la necesidad de completar la información del Catálogo... ampliando el conocimiento de los yacimientos ya censados en los aspectos no trabajados en profundidad. No queremos dejar de advertir aquí que aún necesitamos dirigir los trabajos de catalogación a otras manifestaciones "artísticas", como son los grabados rupestres.

2. Era preciso, pues, confeccionar un trabajo que recabara toda la información necesaria para definir las variables de afección o desafección sobre el patrimonio arqueológico. Para ello deberían analizarse y evaluarse los siguientes parámetros:

- La incidencia sobre los enclaves con manifestaciones de arte rupestre de determinados elementos del planeamiento urbanístico municipal como: la estructura general y orgánica del territorio, calificación y ordenación física, ordenanzas de suelo urbano, normativa de protección, catálogo urbanístico de edificios, ordenanzas concretas para yacimientos situados en suelo no urbano y urbanizable, etc. 
- La incidencia sobre el mismo de las determinaciones y actuaciones derivadas del desarrollo de la legislación medioambiental.

- La incidencia de las determinaciones y actuaciones derivadas de otras figuras de desarrollo de la legislación del Suelo y de Ordenación del Territorio.

- La incidencia de las determinaciones y actuaciones derivadas de planes, programas y proyectos de desarrollo económico, turístico, etc.

- La incidencia de las determinaciones y actuaciones puntuales en la ordenación de los espacios naturales donde se ubiquen los yacimientos.

- Asimismo, era necesario analizar las actuaciones que se habían llevado a cabo en desarrollo de la propia legislación de Patrimonio Histórico.

- Y, por último, no sólo había que documentar la formalización actual de esas determinaciones en el territorio sino también sus tendencias futuras.

Esta información sería básica a la hora de plantear las prioridades de protección que se esbozarán más adelante, y sus resultados vendrían a conformar una auténtica Carta de Riesgo para las cuevas y abrigos con arte rupestre y sus ámbitos de afección.

3. La primera consecuencia del trabajo sería la delimitación de las áreas que conformarán Zonas Arqueológicas declaradas como Bien de Interés Cultural para cada uno de estos enclaves y sus entornos. Expedientes que irán gestionándose en función de la necesidad más o menos apremiante de protección de los yacimientos, a tenor de los datos aportados. Otras conclusiones lógicas serían las de generar los criterios para la elaboración de los informes técnicos preceptivos que desde la Consejería de Cultura se deben emitir tanto a los particulares como a otras administraciones (Rodríguez de Guzmán Sánchez et al. 2002b).

Según estas consideraciones, en el verano de 1997 se publicó en el BOJA el concurso para la contratación del trabajo de consultoría y asistencia técnica denominado Delimitación de las áreas de riesgo de destrucción de los yacimientos con arte rupestre en Andalucía, que comprendía todos los yacimientos de la provincia de Málaga y parte de los de las provincias de Cádiz y Jaén. Y el trabajo se adjudicó bajo las citadas determinaciones.

La propia naturaleza del encargo incitaba a reflexionar sobre algunas cuestiones. Para empezar, había que considerar a las estaciones con arte rupestre desde una perspectiva más amplia que la histórico-arqueológica propiamente dicha pero, al mismo tiempo, no debía olvidarse la problemática que desde este punto de vista aquéllas ofrecen. Así, observamos que estos yacimientos responden a una variada tipología: desde el abrigo de reducidas dimensiones que se abre en una pared rocosa y conserva algunas pinturas o trazos de color o motivos grabados -el Abrigo de la Venta del Fraile en Almogía (Málaga), el Abrigo de Navalcán en Noalejo (Jaén) y los numerosos abrigos del municipio de Jaén, entre otros-adscribibles al denominado fenómeno esquemático, hasta los grandes complejos cársticos que, junto a una impresionante colección de arte prehistórico-abarcando la mayoría de las veces desde el Paleolítico Superior hasta el Calcolítico-conservan potentes niveles de ocupación-cuevas de Doña Trinidad en Ardales o de Nerja, ambas en Málaga. Es posible, incluso, diferenciar un tercer grupo: pequeñas cuevas con arte parietal paleolítico-Cueva del Calamorro (Benalmádena, Málaga) y Cueva de La Araña (Málaga) que también fueron usadas, al parecer simultáneamente, como lugar de habitación-y grandes abrigos con arte esquemático-Cueva de Las Grajas (Archidona, Málaga) y Abrigo de la Higuera (Mollina, Málaga)- cuyas pinturas pueden asociarse a yacimientos arqueológicos que se encuentran en el interior o en las inmediaciones de estas cavidades. En las dos últimas categorías citadas, los niveles arqueológicos soterrados han sufrido, por regla general, un alto grado de destrucción. 
Mas, sin embargo, en ningún caso se trató el arte rupestre primando cuestiones intrínsecas a su génesis y desarrollo-estilo, tipología, técnicas, cronología y/o adscripción cultural-, aspectos directamente vinculados a la investigación histórico-arqueológica que, por otra parte, han sido, en mayor o menor medida, estudiados por diferentes especialistas. Y, pese a haber visitado cada uno de los emplazamientos de las estaciones con arte rupestre, tampoco se realizaron análisis del estado de conservación de las pinturas propiamente dichas y sus posibles soluciones, valoración que no formaba parte de los requerimientos del encargo. No obstante, la información existente en ambos sentidos -investigación y conservación- unida al estudio del territorio, permitió no sólo realizar una valoración del estado actual de cada una de las estaciones con arte rupestre, sino también confeccionar unas medidas específicas de preservación a partir de la distinción de los riesgos, reales y potenciales, que pueden advertirse en estos enclaves arqueológicos (Santana Falcón 2001). ${ }^{5}$

El diagnóstico, ponderando los factores de riesgo de mayor incidencia sobre los yacimientos y el grado de afección que representan en cada uno de ellos, abarca todos los campos de la Tutela del Patrimonio Histórico, y toma en cuenta muy especialmente las posibilidades que tienen estos sitios para soportar su posible apertura al público. Concretando, se definen objetivos en materia de desarrollo de figuras de protección delimitando ámbitos, reducidos en algunos casos y con amplios entornos en otros, que pueden incluir una variada tipología de yacimientos; se aportan propuestas para la creación o desarrollo de unidades administrativas, para la realización de tareas de vigilancia, para los cerramientos y vallados de algunos enclaves y para las cubriciones o enterramientos de antiguas excavaciones arqueológicas; se valora la conveniencia de abordar determinadas líneas de investigación y de adoptar medidas de conservación básica; asimismo, se proponen medidas de difusión y adecuación para la visita, etc.

Nos centraremos ahora en el análisis de las estaciones con arte rupestre del municipio de Jaén, que suman un total de cincuenta sitios englobados en veintiocho emplazamientos. Antes que nada, debemos reseñar las dificultades que tuvimos para ubicar con exactitud algunos yacimientos porque guardan una estrecha relación con cualquiera de las soluciones que finalmente se ofrecieron. Explicaremos algunos ejemplos:

- Estaciones descritas en la bibliografía científica que no es posible localizar si no es mediante una prospección arqueológica intensiva del ámbito donde se encuentran: Peñas de Castro, Cueva Secreta, abrigos y paredes de El Canjorro, del Río Frío y del Cerro del Frontón, publicadas desde mediados de los años ochenta. ${ }^{6}$ Estos sitios se recogen en ARQUEOS y en el catálogo del PGOU de Jaén asignándoseles un área de protección.

- Yacimientos que se citan en la bibliografía científica sin indicaciones concretas sobre su ubicación: Barranco de la Tinaja, Cerro Veleta y Peña de la Salada. También se encuentran en ARQUEOS, pero no en el planeamiento urbanístico.

- Existen otros sitios con arte rupestre en la comarca aún inéditos: pinturas y grabados de la Serrezuela en Pegalajar, sin indicación concreta de su ubicación, y el abrigo de Peñas Rubias, que no sabemos con exactitud en qué municipio se encuentra.

La recopilación bibliográfica permitió unificar documentación gráfica y cartográfica y contar con plantas y secciones de los enclaves, de gran ayuda para delimitar las áreas de protección y establecer la normativa que debe acompañarlas, sobre todo cuando hay enclaves que no logramos visitar por las causas que explicamos antes. Pero la información gráfica para los yacimientos de Jaén era bastante escasa: las reproducciones de los paneles, totales o parciales - cueva del Plato-, no superan la media docena sitios; a veces se trata de

5. En el trabajo citado se explica la metodología empleada, las características de los documentos elaborados y las distintas partes de que constan cada uno de ellos.

6. Véase la bibliografía citada. 
dibujos que tienen ya más de veinte años -Abrigo de la Cantera-y que sería necesario revisar; en otros casos, las reproducciones de la publicación ofrecen tan poco detalle, o son tan pequeñas, que preferimos no incluirlas: Abrigo de los Herreros. Ningún abrigo cuenta con un buen levantamiento topográfico, actividad que reclamamos se emprenda con la mayor urgencia, a excepción de las plantas y secciones de los abrigos del Plato y de la Higuera.

Con respecto al análisis de la documentación urbanística, diremos que el Plan General de Ordenación Urbana de Jaén fue aprobado definitivamente el 26 de febrero de 1996. Este documento concede un tratamiento específico al Patrimonio Arqueológico y cuenta con un catálogo de yacimientos, dieciocho de los cuales son estaciones con arte rupestre. ${ }^{7}$ Todas los sitios de estas características se encuentran en suelo calificado como No Urbanizable y clasificado como sigue:

* Suelo No Urbanizable Común

* Suelo No Urbanizable Protegido en base a su inclusión en el Catálogo de Espacios Protegidos del Plan Especial de Protección del Medio Físico

Las Condiciones Generales de Protección para el Suelo No Urbanizable Común distinguen, entre otras de menor interés para nosotros, la protección del medio ambiente que, en algunos extremos, podemos, y debemos, relacionar directamente con la de los yacimientos arqueológicos, especialmente en lo referido a la protección del paisaje. Aquí se incluyen cuestiones como la instalación de vertederos, de depósitos de vehículos y de chatarra, de elementos de publicidad y los proyectos de construcción de imágenes o símbolos en las cumbres de los montes y otros lugares especialmente visibles. Todos los proyectos de estas características deben someterse a diversas autorizaciones administrativas que en ningún caso incluyen a la Consejería de Cultura.

Para los yacimientos arqueológicos en suelo no urbanizable se concretan también unas normas de protección específicas que establecen usos compatibles y prohibidos, remitiendo directamente al régimen específico del Suelo No Urbanizable Protegido por Paraje Natural Excepcional.

Sin embargo, según el régimen urbanístico del SNU Común, y la normativa particular establecida a este respecto, una vez obtenidos los correspondientes informes favorables en los suelos así calificados es posible realizar casi cualquier actividad, ${ }^{8}$ incluso proyectar distintos tipos de instalaciones y edificaciones. Esta realidad, que conlleva un elevado riesgo de destrucción para el Patrimonio Arqueológico, se ve atenuada al especificar que si se detectara un yacimiento en áreas cuyas determinaciones urbanísticas no resultasen adecuadas con su preservación, éstas quedarán -previa decisión del Organismo o Entidad competenteautomáticamente sujetos a la suspensión cautelar de las autorizaciones, licencias y permisos para actuar en tanto no se apruebe la necesaria modificación del Planeamiento, otorgando así exclusivamente a la Consejería de Cultura la capacidad de decidir en este ámbito.

Los usos que se reconocen como característicos del suelo no urbanizable son los que se adapten a las particularidades naturales de este suelo; y los usos compatibles aquellos que, previa licencia municipal y, en su caso, autorización de la Comisión Provincial de Urbanismo, puedan implantarse siempre y cuando se acredite específicamente que el impacto producido es admisible en relación con las normas de carácter general establecidas para cada categoría de suelo no urbanizable. En resumen, una lectura detenida de este capítulo concluirá que, al igual que sucedía con el régimen urbanístico, una vez obtenidas las autorizaciones que para cada caso se prescriben, el Suelo No Urbanizable de Jaén puede acoger actuaciones urbanísticas

7. Los yacimientos de Peñas Rubias, Cerro Veleta y Peña de la Salada no se recogen en el planeamiento ni, como explicamos antes, pudimos localizarlos a partir de las datos con que contábamos.

8. En el SNU Común de Jaén pueden llegar a realizarse las siguientes actividades: infraestructurales, extractivas, relacionadas con la explotación de recursos vivos, industriales, turísticas y recreativas y vertederos. 
de cualquier índole. Y aunque los yacimientos arqueológicos estén sometidos a un régimen más restrictivo, ${ }^{9}$ este instrumento podría perder eficacia en delimitaciones tan concretas como las recogidas en el P.G.O.U.

El Suelo No Urbanizable Protegido se clasifica en varios tipos; los que interesan a este trabajo son los siguientes: Paraje Natural Excepcional (SUNP PE), Área forestal de Interés Recreativo (SUNP FR) y Yacimiento de Interés Científico (SNUP YA).

Los dos primeros son la transposición del régimen de protección previsto en las Norma 32, Parajes Naturales Excepcionales, y 38, Espacios Forestales de Interés Recreativo, del Plan Especial de Protección del Medio Físico de la Provincia de Jaén, y en sus delimitaciones engloban a la gran mayoría de estaciones con arte rupestre del Término Municipal. Las determinaciones específicas de protección para el suelo no urbanizable donde exista un yacimiento de interés científico consisten en la asunción de la Norma 34, Yacimientos de interés científico, del P.E.P.M.F. ${ }^{10}$

Figura 1. Estaciones con arte rupestre del municipio de Jaén recogidas en el SIPHA y normativa urbanística que las afecta.

\begin{tabular}{|l|l|}
\hline \multicolumn{1}{|c|}{ Denominación } & \multicolumn{1}{|c|}{ Figura de Protección } \\
\hline ABRIGO DE LA CANTERA & \\
ABRIGO DE LA HIGUERA & \\
ABRIGOS DEL BARRANCO DEL ESTORIL & \\
ABRIGOS DEL BARRANCO DE LA TINAJA & Plan Especial de Protección del Medio Físico. Provincia de Jaén: \\
ABRIGOS DEL CERRO DEL FRONTÓN & Área Forestal de Interés Recreativo Quiebrajano. \\
CUEVAS DE LOS HERREROS & Plan General Municipal de Ordenación Urbana de Jaén \\
CUEVAS DEL PlATO & \\
CUEVA DE LOS SOLES & \\
ABRIGO DEL POYO DE LA MINA & \\
ABRIGO DE LA CANTERA & \\
ABRIGO DE LA HIGUERA & \\
\hline ABRIGO DEL ALMENDRO & \\
ABRIGOS DE LA DIOSA & P.E.P.M.F. Provincia de Jaén: Paraje Natural Excepcional Río Frío. \\
ABRIGO DE LAS PALOMAS & Plan General Municipal de Ordenación Urbana de Jaén \\
CUEVA DE LOS MOLINOS & \\
CUEVA DE RÍO FRÍO & \\
ABRIGO DEL POYO DE BERNABÉ & \\
ABRIGO DEL POYO DE LOS MACHOS & \\
ABRIGO DEL ALMENDRO & \\
ABRIGOS DE LA DIOSA & P.E.P.M.F. Provincia de Jaén: \\
\hline ABRIGOS DEL CERRO DE LA LLANA \\
ABRIGOS DE LA FUENTE DE LA PEÑa & Área Forestal de Interés Recreativo Jabalcuz y Sierra de la Grana. \\
ABRIGOS DE LA MELLA & Plan General Municipal de Ordenación Urbana de Jaén \\
\hline
\end{tabular}

9. El P.G.O.U. de Jaén no establece diferentes categorías de protección en razón de las especificidades de los sitios arqueológicos, ni siquiera para aquellos, como las estaciones con arte rupestre, declarados Bien de Interés Cultural. La obligatoriedad de redactar un Plan Especial de Protección ante una posible afección a un yacimiento se amplía a todos los sitios arqueológicos.

10. El único yacimiento arqueológico del municipio de Jaén que se incluyó en aquel documento fue el asentamiento ibérico de Puente Tablas. 


\begin{tabular}{|l|l|}
\hline \multicolumn{1}{|c|}{ Denominación } & \multicolumn{1}{|c|}{ Figura de Protección } \\
\hline $\begin{array}{l}\text { ABRIGOS DE LAS PEÑAS DE CASTRO } \\
\text { CUEVAS DE EL CANJORRO } \\
\text { CUEVA SECRETA }\end{array}$ & Plan General Municipal de Ordenación Urbana de Jaén \\
\hline
\end{tabular}

Todos los sitios citados en la figura 1, excepto los abrigos de las Palomas y de la Diosa, el Poyo de Bernabé y el Barranco de la Tinaja, se representan en la planimetría urbanística bajo la categoría Yacimiento de Interés Científico, asumiendo las prescripciones de protección correspondientes. El catálogo de yacimientos arqueológicos del PGOU tampoco detalla los distintos abrigos con arte rupestre del complejo cavernícola de El Canjorro -cuevas Sur, Centro, Oeste y Este-aunque el área de protección grafiada comprende todo el monte desde la cota 650 hacia arriba, ni recoge los enclaves del Cerro Veleta, Peña de la Salada y Abrigo de Peñas Rubias, de los que tampoco pudimos conocer su ubicación.

Por otra parte, se observaron importantes discrepancias entre algunas coordenadas que se relacionan en Arqueos y su plasmación en la planimetría urbanística, como se puso de manifiesto, por ejemplo, en las localizaciones de los abrigos y paredes con pinturas del río Frío, donde, además, la representación gráfica de polígonos que se superponen dificulta la apreciación de los distintos ámbitos delimitados.

Pues bien, tomando en cuenta las consideraciones expuestas respecto al Plan General de Ordenación Urbana de Jaén y al Plan Especial de Protección del Medio Físico de la provincia, las peculiaridades físicas y territoriales de las estaciones con arte rupestre, los datos facilitados por la bibliografía especializada, los factores de riesgo que establecimos y el grado de afección que cada uno de ellos representa para estos yacimientos arqueológicos y, por último, las propuestas de actuación de la Delegación Provincial en Jaén de la Consejería de Cultura, ${ }_{11}$ formulamos la siguiente propuesta para la protección de las Estaciones con Arte Rupestre del Municipio de Jaén:

* Solicitar una modificación puntual del Plan General de Ordenación Urbana de Jaén en orden a incluir las nuevas delimitaciones que aportamos, las cuales en todos los casos se dotaron también de un entorno de protección.

* Iniciar la redacción de los expedientes de declaración como Zona Arqueológica B.I.C. a favor de cada uno de los enclaves delimitados, atendiendo especialmente a las particularidades territoriales que ofrecen. Sugerimos que podrían confeccionar tres expedientes:

- Expediente de declaración como Zona Arqueológica Bien de Interés Cultural a favor de las estaciones con arte rupestre de La Mella: La Mella, Fuente de la Peña y La Llana.

- Expediente de declaración como Zona Arqueológica Bien de Interés Cultural a favor de las estaciones con arte rupestre de Peñas de Castro, El Canjorro y del río Frío.

- Expediente de declaración como Zona Arqueológica Bien de Interés Cultural a favor de las estaciones con arte rupestre del valle del Quiebrajano.

La identificación sobre el territorio de áreas de actividad en cuanto a la representación de motivos pintados y grabados es uno de los hilos conductores de nuestra propuesta de protección. A diferencia de otros casos ya estudiados por nosotros en Andalucía, en los lugares donde se encuentran pinturas y grabados no se conocían otros yacimientos arqueológicos que pudiéramos suponer sincrónicos más allá del dolmen de Otíñar y la Cueva de los Corzos, así como materiales dispersos en las inmediaciones de algún abrigo, caso del Abrigo de la Cantera. ${ }^{12}$

11. Nos referimos al expediente para la inscripción específica en el Catálogo General del Patrimonio Histórico de Andalucía de la Zona Arqueológica de Otíñar. Archivo del Servicio de Protección de la Dirección General de Bienes Culturales.

12. Recientemente se ha publicado la existencia de un asentamiento cercano y quizás relacionado con el dolmen de Otínar, del que no teníamos noticias cuando realizamos nuestro trabajo (Zafra de la Torre 2000). 
Las estaciones con arte rupestre de Jaén se hallan en las alineaciones montañosas que se extienden desde el Suroeste de la ciudad en dirección Sur-Sureste, ámbito en el que se han distinguido varios espacios merecedores de especial protección urbanística en base a su singularidad medioambiental y paisajística. La relación entre yacimiento arqueológico, territorio y paisaje se hace muy evidente porque hablamos de una calidad paisajística en algunos lugares insuperable -el valle del Quiebrajano o los cañones del río Frío-, porque la actividad antrópica es casi inexistente o se limita al aprovechamiento del suelo: olivares, pasto para el ganado... Pero, al mismo tiempo, se trata de un paisaje muy vivido, que se percibe en gran parte desde la misma ciudad, o que está sólo a quince minutos en coche -el Quiebrajano es en realidad un parque periurbano; Jabalcuz es tradicionalmente zona de segunda residencia para los giennenses. Y justamente de todo ello derivan las amenazas más graves para el patrimonio arqueológico.

El macizo de Jabalcuz y Sierra de la Grana, el más septentrional, alberga los abrigos de La Mella, del Cerro de La Llana y de la Fuente de la Peña que engloban un total de doce estaciones. Optamos por establecer una delimitación particular como Bien de Interés Cultural para cada uno de los emplazamientos citados, ampliando en el primer caso el polígono establecido en el PGOU a toda la cresta rocosa donde se encuentran las pinturas, y modificando los polígonos que recoge el planeamiento en los otros sitios.

Con respecto al entorno de protección, diferenciamos el conjunto que representan las pinturas de $\mathrm{La}$ Mella y del cerro de la Llana, ubicadas en las vertientes oeste y sur del cerro de los Morteros (Figura 2), con respecto a los abrigos del Cerro de la Fuente de la Peña.

La delimitación se sustenta, además de lo apuntado más arriba en cuanto al proceso de urbanización que padece la zona, en la necesidad de impedir otros usos igualmente incompatibles con la preservación de los yacimientos, por ejemplo la implantación de aerogeneradores que ya ha sido solicitada para la Sierra

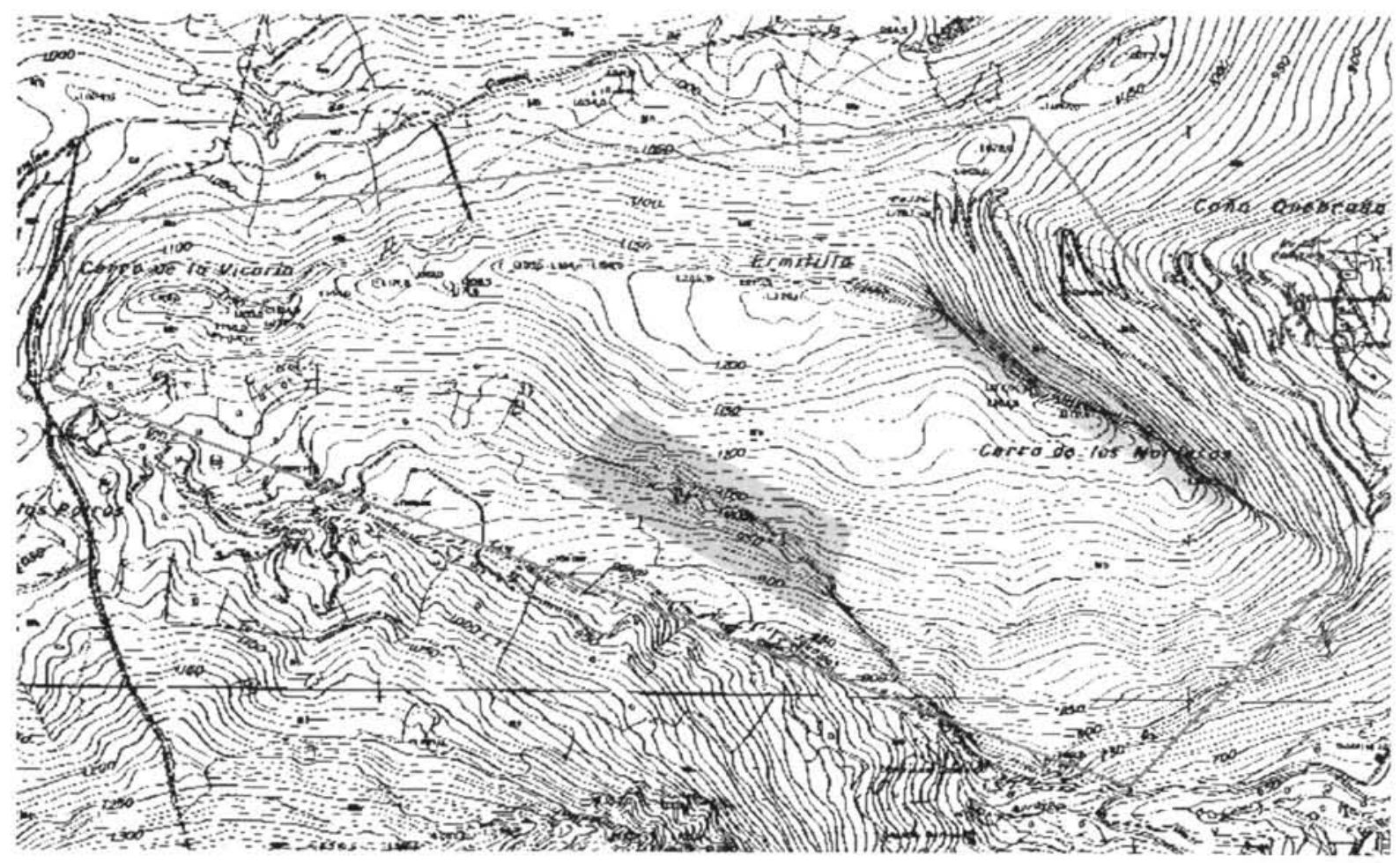

Figura 2. Propuesta de delimitación de la Zona Arqueológica B.I.C. La Mella. Más oscuras la propuesta de delimitación de los bic's. La Mella (arriba, a la derecha) y Cerro de la Llana (abajo, al centro). Polígono gris claro, propuesta de delimitación del entorno. E. 1:20.000. 
de Jabalcuz. Por último, la posibilidad de que los innumerables abrigos y paredes susceptibles de albergar restos pictóricos que salpican toda la zona contengan otras manifestaciones artísticas, también aconseja establecer un ámbito de protección amplio que limite actividades claramente perjudiciales para su preservación; actividades que, por otra parte, ya se regulan en el P.G.O.U. atendiendo las determinaciones del Plan Especial de Protección del Medio Físico de la Provincia de Jaén, aunque en unas condiciones menos restrictivas que las propuestas por nosotros y considerando un ámbito de afección mucho menor para los yacimientos arqueológicos.

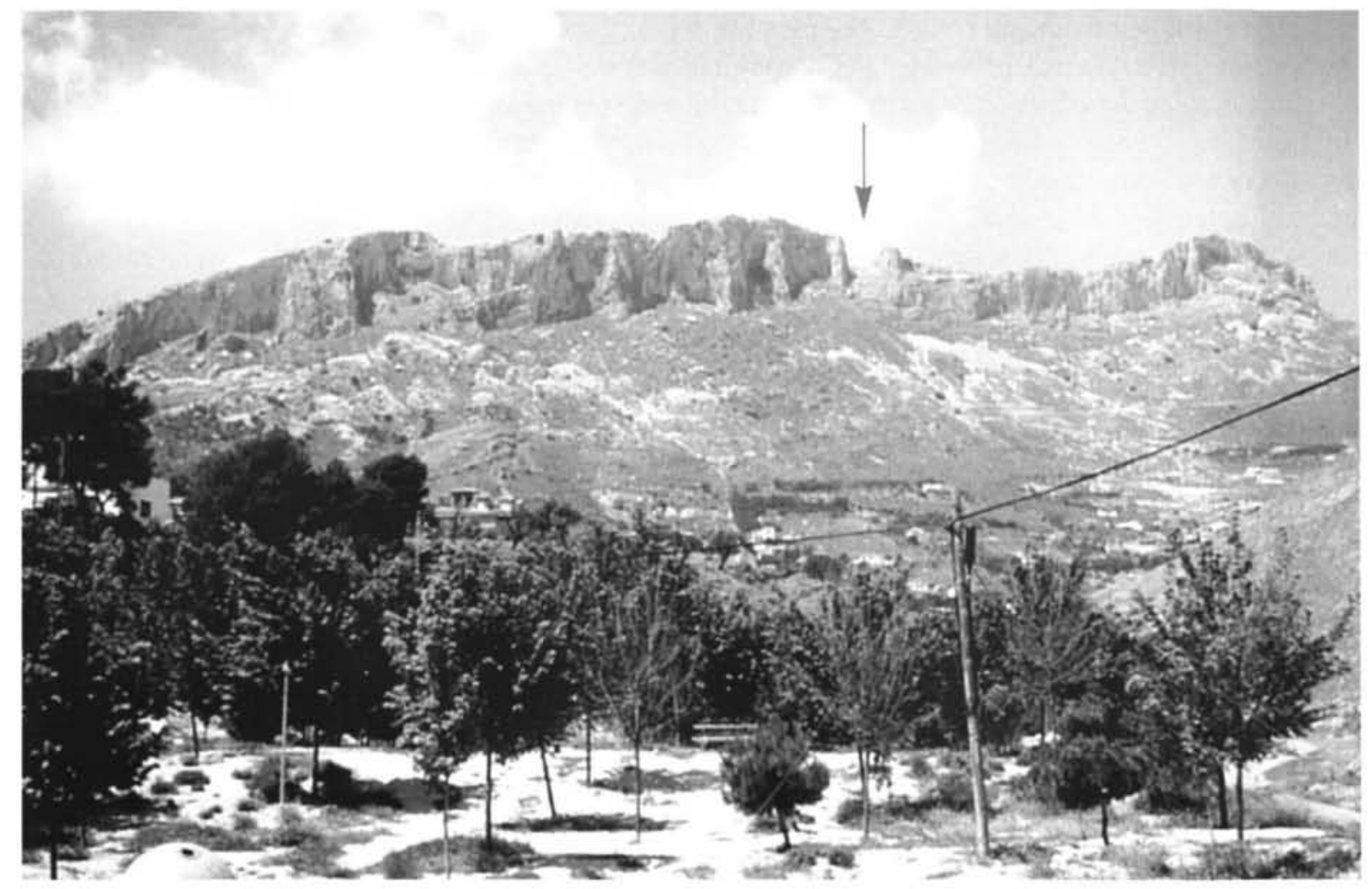

Figura 3. Vista general de La Mella.

Las pinturas rupestres del cerro de la Fuente de la Peña están afectadas sobre todo por infraestructuras de variada índole, que se mantienen en servicio, y por la existencia de una cantera de caliza, ahora fuera de uso. A pesar de que no se observa el fenómeno de expansión urbanística al que nos referíamos antes, las servidumbres de este cerro no deben menospreciarse porque los trabajos de mantenimiento de infraestructuras nuevas y/o complementarias podrían incidir muy negativamente en la preservación del arte rupestre.

Al Sureste de este conjunto se encuentran las Peñas de Castro y la garganta del río Frío, que albergan varias estaciones con arte rupestre. En las Peñas de Castro se conocen cuatro abrigos bajo esa denominación así como una gran grieta que contiene también pinturas rupestres, Cueva Secreta. Este enclave continúa hacia el Sur, estrechándose y perdiendo altura hasta la garganta del río Frío, mediante un cerro-espolón, Canjorro, salpicado de pequeñas cavidades con pinturas y otros indicios de ocupación prehistórica. Al río, en el PGOU área de protección especial por Paraje Natural Excepcional, se asoman también varios abrigos con pinturas: de la Diosa, de las Palomas, del Almendro..., y algunas de las paredes de la garganta también poseen pinturas rupestres: Poyo de los Machos, de Bernabé, etc. 


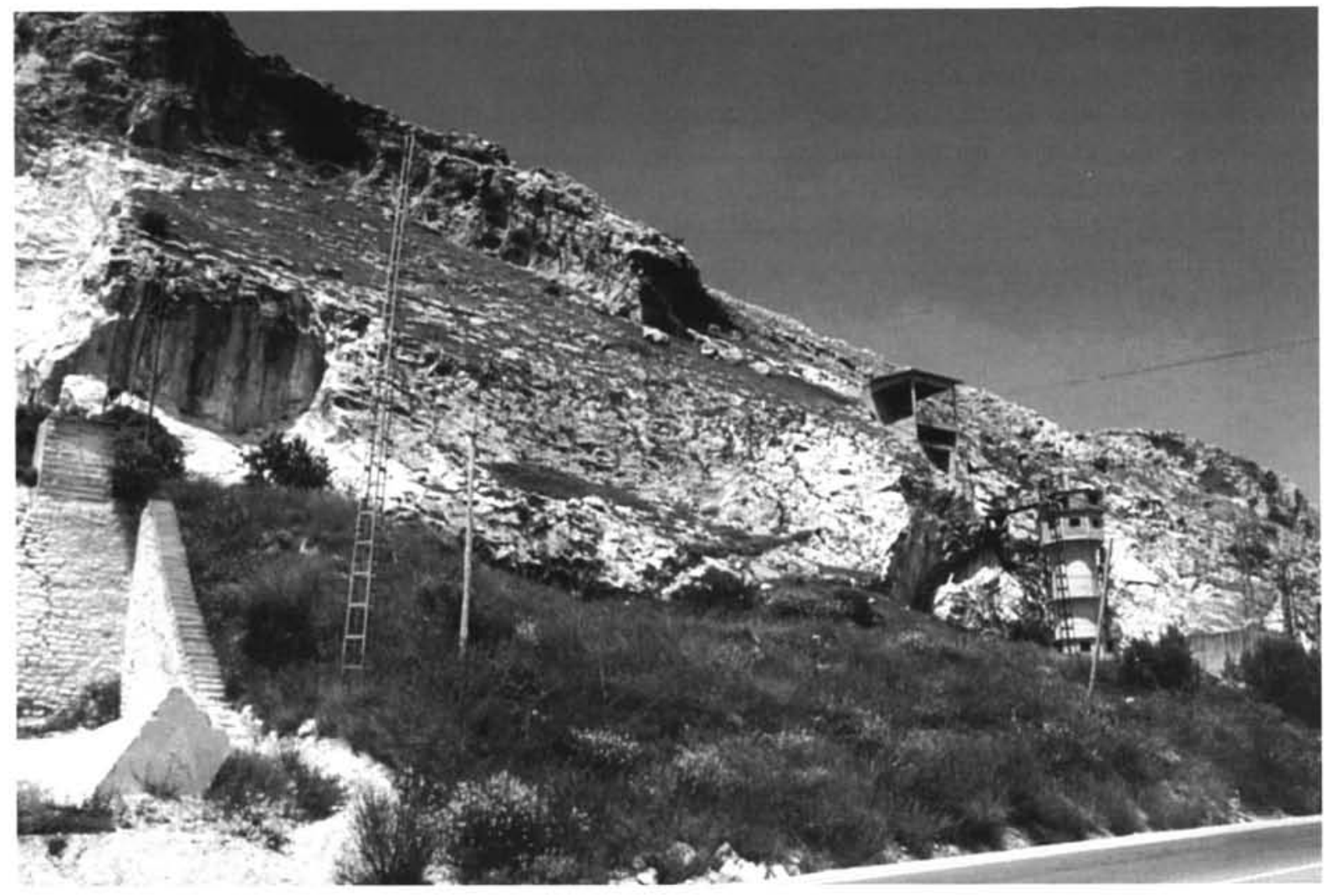

Figura 4. Cerro Fuente de la Peña.

Toda la zona, que debió ser habitada y utilizada por el hombre durante la Prehistoria postpaleolítica como indica el número y dispersión de los yacimientos arqueológicos descubiertos, goza de diferentes ámbitos de protección en razón de la existencia de los abrigos con arte rupestre, con la excepción de los de El Canjorro englobados en un único polígono. No obstante, Peñas de Castro y Canjorro se clasifican en el planeamiento como Suelo No Urbanizable Común, y no gozan de protección específica en el P.E.P.M.F. Por ello, a pesar de que la vocación del suelo en esta zona es agrícola, y de hecho se dedica a olivar, nos pareció aconsejable delimitar un entorno amplio que defendiera a los yacimientos de actuaciones urbanísticas futuras que hagan peligrar su integridad.

En base a estas circunstancias pensamos que, sobre todo, era necesario ordenar diferentes ámbitos de protección. Partimos de los emplazamientos que propone el PGOU para sugerir varias modificaciones:

* Peñas de Castro. Tanto para Cueva Secreta como para los abrigos 1-4 de Peñas de Castro mantenemos los polígonos que delimitan el bien, añadiendo un amplio entorno que va de norte a sur a lo largo de toda la línea de escarpes y abarca de oeste a este las dos vertientes.

* El Canjorro y Río Frío (Figura 5). Establecimos dos ámbitos para la Zona Arqueológica B.I.C.: uno para la cueva de los Molinos y otro para el resto de los yacimientos. Este último avanza, de oeste a este, desde la ladera occidental del Canjorro a lo largo de la garganta del río Frío. El límite norte se establece en la estribación meridional de las Peñas de Castro para incluir el único paso existente hacia la cara oeste del Canjorro. El límite sur se mantiene en paralelo a los cañones del río Frío abarcando las paredes meridionales de la garganta. 


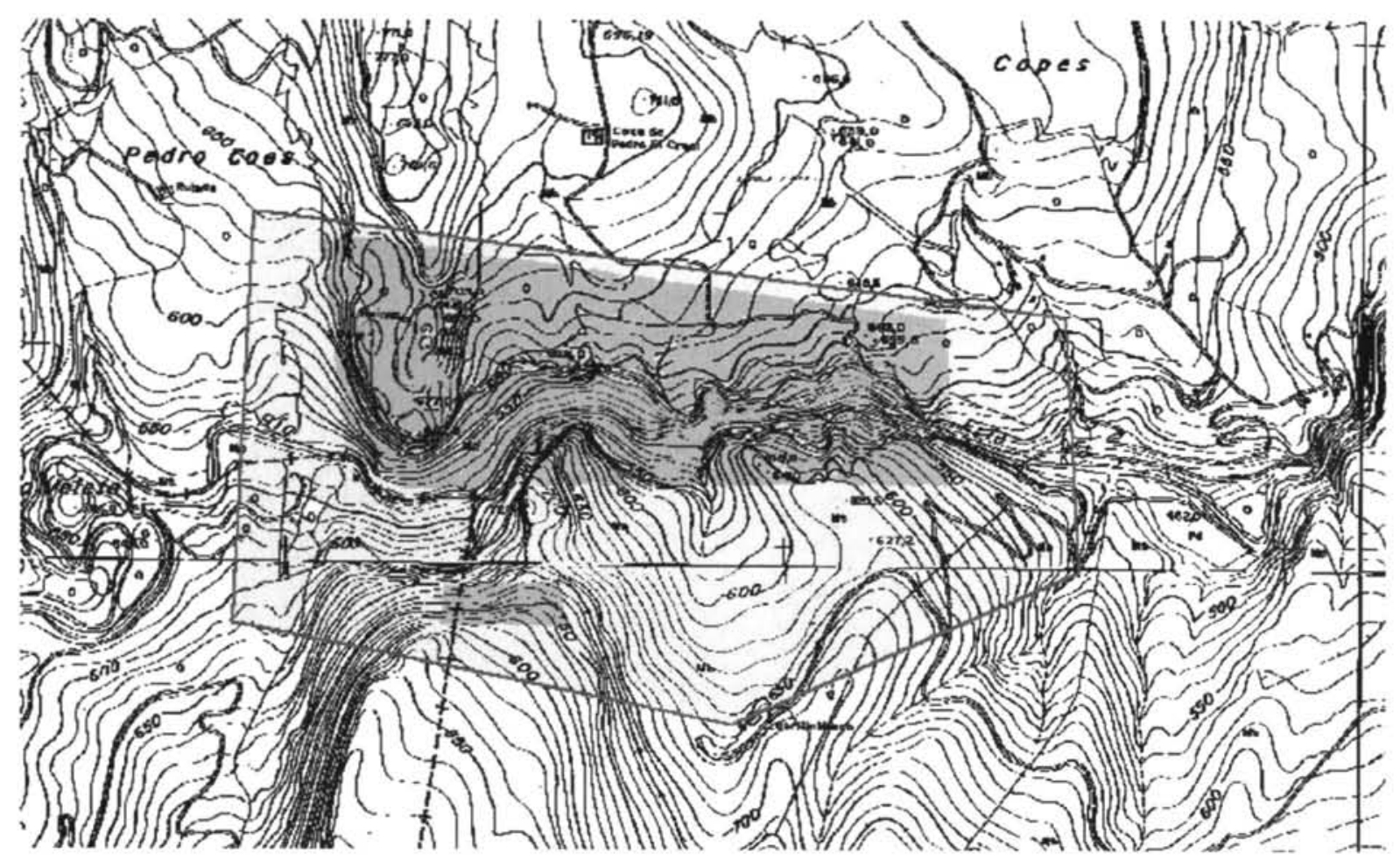

Figura 5. Propuesta de protección para las estaciones con arte rupestre de El Canjorro y río Frío. Los polígonos de propuesta de delimitación de los bic's en tono más oscuro; más claro, la propuesta de delimitación del entorno. E. 1:20.000.

Este área engloba con holgura los polígonos representados en el documento urbanístico y establece la zona susceptible de protección desde un punto de vista dinámico, tomando en consideración la existencia de una actividad humana que no se limita a lugares con pinturas y grabados. Lugares que, por otra parte, se ordenaron en un único ámbito de protección.

El entorno lo mantuvimos hacia el norte en torno a los 600-700 $\mathrm{m}$ de altitud. Por el sur, se hizo coincidir con la delimitación del Paraje Natural Excepcional Río Frío, adaptándose el extremo occidental al inicio de la garganta por donde circula el río y recogiendo todo el frente del escarpe donde se encuentran la abrigo del Almendro, el Poyo de Bernabé, los abrigos de la Diosa, etc. Desde aquí, término municipal de Los Villares, avanza hacia el norte subiendo hasta encontrar el vértice noroeste del polígono. Hacia el este, el límite sur recoge el camino serpenteante que siguiendo el curso del río conduce a los cañones -al final de este camino se encuentra una de las depuradoras de agua que abastecen a la ciudad de Jaén, por lo que no es posible acceder al río por este lugar. El límite oriental lo llevamos a las vaguadas de escorrentía que se aprecian en ambas márgenes del Frío.

Para la Cueva de los Molinos modificamos el polígono inicial, ampliándolo para acoger toda la pared donde se ubica el abrigo.

El valle del Quiebrajano alberga un total de quince estaciones con arte rupestre, además de otros elementos patrimoniales de interés como un enterramiento dolménico, la cueva de los Corzos, una villa romana, el castillo y el caserío de Otínar, los cuales, a excepción del primero, no incluimos áreas de protección. En lo que respecta a las estaciones con arte rupestre, se diferencian dos grandes ámbitos, que describimos de norte a sur: 


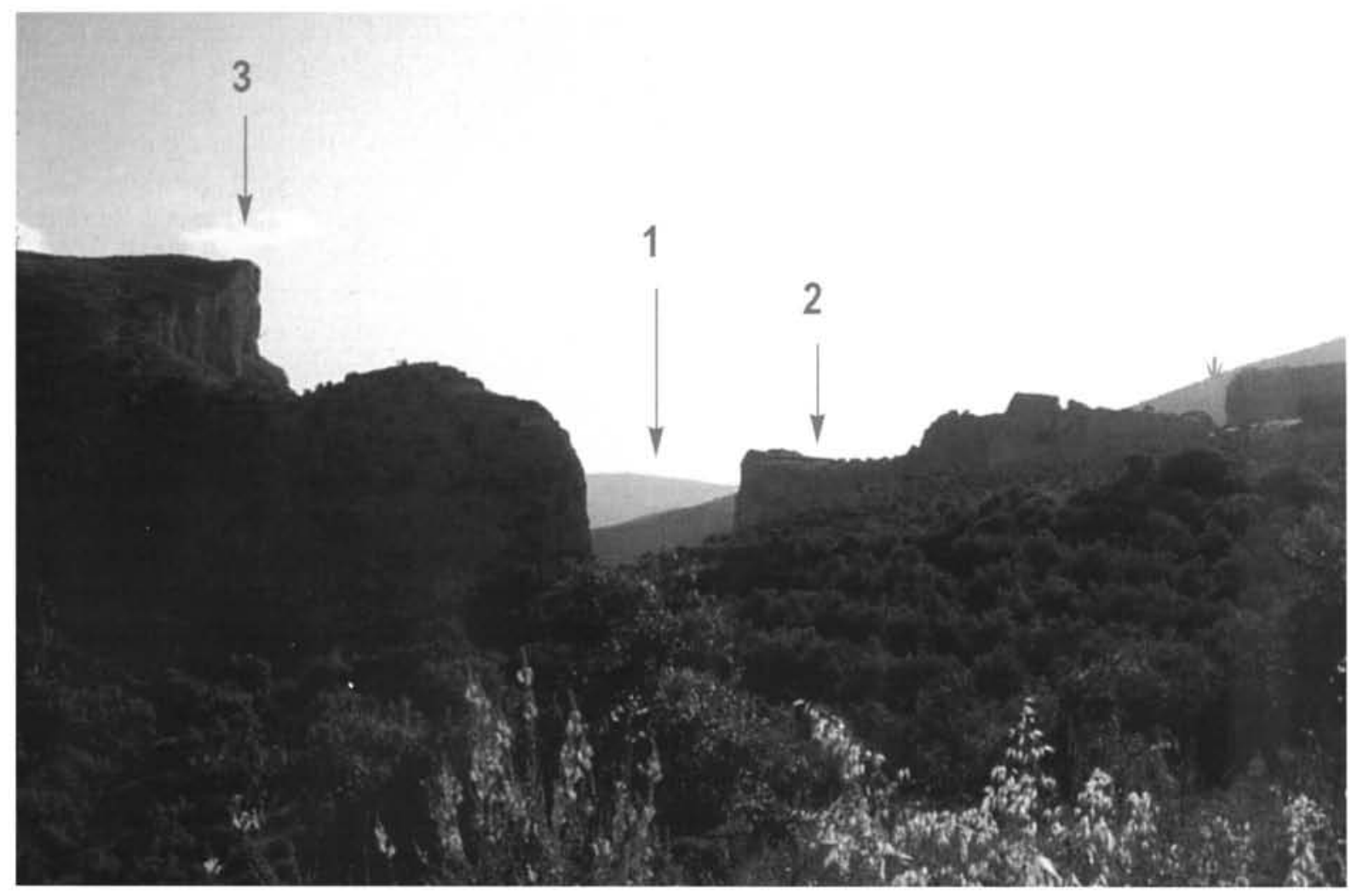

Figura 6. 1. Cañón de Río Frío 2. Cerro Canjorro 3. Emplazamiento de la cueva de Los Molinos.

* Quiebrajano I. Estaciones con arte rupestre del Cerro del Frontón, Cerro Veleta y La Bríncola. Establecimos una delimitación del bien para cada sitio. El correspondiente al cerro Veleta recoge los abrigos con arte rupestre de los Soles, Poyo de la Mina y Herreros, así como el dolmen de Otíñar. La delimitación del B.I.C. de La Bríncola incluye los abrigos de la Higuera y del Plato. El Cerro del Frontón acoge cuatro estaciones con arte rupestre bajo esa denominación.

En todos los casos modificamos, ampliándolas y adaptándolas al territorio, las delimitaciones del planeamiento. El caso más evidente quizás sea el cerro Veleta, donde el área de protección propuesta incluye el espolón que avanza hacia el Quiebrajano. En ella incluimos los abrigos de los Herreros, poyo de la Mina y cueva de los Soles, así como el dolmen de Otíñar, que evidencia la diversidad de usos del territorio desde la Prehistoria. Para los abrigos del Plato y de la Higuera diseñamos un polígono que ocupa la pendiente en la que se ubican a lo largo de casi 200 metros de longitud. En el Cerro del Frontón variamos ligeramente el área de afección que estima el PGOU para incluir la totalidad del cerro.

La visita a cada uno de los emplazamientos citados nos hizo notar la estrecha relación visual que se establece entre ellos, todos en cotas altas y destacadas y siempre asomándose al curso del Quiebrajano, y con respecto a otros elementos geográficos igualmente notorios del entorno inmediato, principalmente el Cerro Calar y el Cerro del Frontón. En función de esta relación y tomando en cuenta, como dijimos, la sincronía cultural de todos los elementos patrimoniales que debíamos considerar, optamos por establecer un generoso entorno de protección que, desde las cotas más altas del Cerro Calar -donde pueden haber otros restos pictóricos a tenor de la bibliografía consultada-, avanza hasta cruzar la garganta del Quiebrajano 
para enlazar en La Bríncola con los abrigos de la Higuera y del Plato. Desde aquí vuelve a cruzar el río para, desde los pies del cerro Veleta, prolongarse hasta el Cerro del Frontón al cual recorre hacia el oeste, desde donde gira al sur englobando la falda del cerro Calar. La expansión del polígono hacia al oeste y suroeste tiene en cuenta la gran cantidad de abrigos de variadas dimensiones y disposición que se abren en estas paredes, los cuales, que sepamos, no han sido prospectados con metodología arqueológica.

* Quiebrajano II. Estaciones con arte rupestre del Abrigo de la Cantera, Barranco de la Tinaja y Barranco del Estoril. Las dificultades de localización del primero aconseja ampliar el ámbito de afección que recoge el P.G.O.U. para englobar en él toda la pared donde al parecer se encuentran las pinturas. Con respecto al abrigo del barranco del Estoril y paredes adyacentes, mantenemos la delimitación inicial. Para las pinturas del barranco de la Tinaja decidimos establecer una nueva delimitación del bien, coincidente con el lugar que indican las coordenadas de ARQUEOS.

El límite oriental del entorno lo establecimos entre la aldea de Santa Cristina y el castillo de Otíñar, desde donde se prolonga hacia el suroeste siguiendo el camino de acceso a la aldea y ascendiendo por la pendiente para encontrar el camino que desde la aldea de Santa Cristina va hacia el castillo; transcurre paralelo a aquél hasta su vértice más occidental, donde gira para encontrar el Barranco de la Tinaja. La delimitación propuesta no valora el entorno de protección que deberá considerarse para el Castillo de Otínar, porque analizar tal cuestión quedaba fuera de los límites de este trabajo.

Porque somos conscientes de la gran extensión de terreno que abarcan estas propuestas es necesario recordar que se encuentra en su totalidad dentro del Área Forestal de Interés Recreativo Quiebrajano, sometida a distintas protecciones urbanísticas que la delimitación como Zona Arqueológica Bien de Interés Cultural sólo vendrá a incrementar con propuestas concretas y, especialmente, regulando, a través de informe preceptivo y vinculante de la Consejería de Cultura, determinadas actividades permitidas o permisibles en la legislación sectorial. Y es que, a nuestro entender, cada uno de las zonas arqueológicas descritas debe recibir un tratamiento unitario a la hora de definir y determinar una protección efectiva, centrada exclusivamente en los yacimientos arqueológicos y, en todo caso, aún más restrictiva que la actualmente impuesta por los documentos urbanísticos analizados.

Para el caso de los abrigos con arte rupestre del valle del Quiebrajano, la solución que ofrecemos varía sustancialmente del Expediente de Inclusión con carácter específico como Zona Arqueológica de Otíñar en el Catálogo General del Patrimonio Histórico de Andalucía, redactado por la Delegación Provincial de Jaén. Este documento parte del Señorío de Otíñar como ámbito de actuación principal, adoptando los que fueron sus límites catastrales para definir el área de afección de la zona arqueológica; límites en los que quedan incluidos una serie de yacimientos de distintas tipologías y cronologías cuyas áreas de afección se grafían siguiendo los mismos criterios que en el Plan General de Ordenación Urbana.

Nosotros no observamos el territorio desde el punto de vista de su habitación continuada por el hombre, constatada sin solución de continuidad desde la Prehistoria postpaleolítica-Cueva de los Corzos, pinturas y grabados rupestres, villa romana del Laurel, castillo, monolito de Carlos III y aldea de colonización de Santa Cristina. Desde esa óptica vemos un sitio histórico de gran entidad y una enorme carga patrimonial, cuya resolución en un expediente administrativo debería ser precisamente esa, la declaración como Sitio Histórico. Ello permitiría, entre otras cuestiones, albergar delimitaciones puntuales de zonas arqueológicas y sus respectivos entornos.

Pues bien, mirando al valle del Quiebrajano desde los emplazamientos del arte rupestre, distinguimos dos grandes concentraciones: al norte, Quiebrajano l, cinco sitios que suponen al menos siete lugares con pinturas y grabados. Éstos, auténticos hitos paisajísticos situados en puntos muy destacados, ocupan los dos márgenes de la garganta -estaciones del cerro Veleta y de la Higuera y del Plato- o bien desde ellos se controlan visualmente unos emplazamientos u otros - Cerro del Frontón/Cerro Veleta/Cuevas de la Higuera y del Plato (Figura 7). 


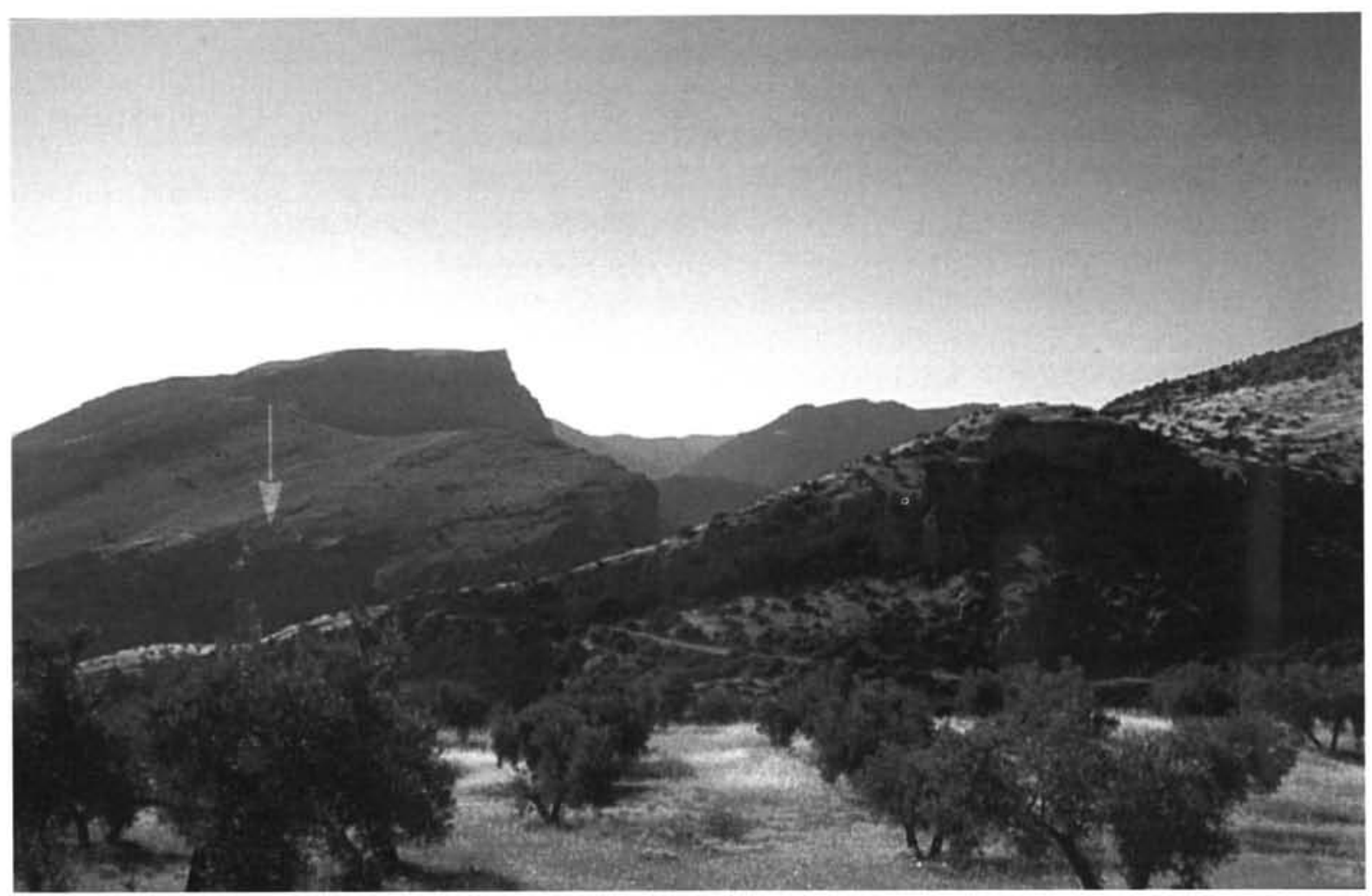

Figura 7. En primer plano, falda del cerro Veleta donde se encuentran el Poyo de la Mina y el dolmen de Otíñar. Al otro lado del río, indicada con la flecha, localización de los abrigos de la Higuera y del Plato.

$\mathrm{Al}$ sur, el conjunto de abrigos y paredes con pinturas y grabados que denominamos Quiebrajano II, representa un caso similar, aunque a menor escala.

El otro elemento a considerar por nosotros a la hora de plantear áreas susceptibles de protección, como bien o como entorno, tiene un carácter mucho más pragmático, tanto como lo son la clasificación y calificación urbanística del suelo, los usos actuales del mismo y los que, a tenor de las circunstancias que afectan a cada uno de los enclaves con arte rupestre prehistórico, podemos presumir que se plantearán en un futuro más o menos inmediato.

De la combinación de ambas magnitudes surgió nuestra propuesta de protección, la cual dentro del orden de prioridades de actuación quizás debiera acometerse en primer lugar habida cuenta de las presiones urbanísticas que se vienen ejerciendo en distintos lugares, como La Mella y el Cerro de la Llana.

Sería también necesario plantear una modificación del PGOU de Jaén en orden a recoger los nuevos ámbitos de protección que proponemos, y las coordenadas que para cada uno de ellos y sus entornos adjuntamos, así como para clarificar el catálogo de yacimientos en lo que se refiere a incluir o modificar algunas denominaciones de las estaciones con arte rupestre, reconociendo además para cada una de ellas su condición de Bien de Interés Cultural.

La siguiente línea de actuación proviene del ámbito de la conservación de los bienes culturales. Aquí, en realidad, se entremezclan intervenciones de diversa índole que van desde la localización topográfica exacta de cada uno de los sitios hasta la redacción de proyectos de cerramiento, pasando por el análisis del estado actual de las manifestaciones artísticas para determinar las medidas más convenientes de cara a su mejor conservación. Siempre tuvimos presente dos cuestiones, desde nuestro punto de vista muy 
importantes: la irreversibilidad de los procesos de deterioro, que para algunos sitios deberemos asumir sin complejos; y el enorme valor del paisaje en la compresión de estas manifestaciones artísticas, paisaje en el que están perfectamente integrados pinturas y grabados, pero muy susceptible a la introducción de elementos extraños, aunque necesarios tal vez para proteger las cavidades.

Así, apostamos por llevar a cabo un proyecto de reproducción gráfica y fotográfica de las pinturas y grabados realizado por profesionales de la imagen supervisados por un arqueólogo especialista en arte rupestre; puede dar espléndidos resultados sin agredir las manifestaciones artísticas. También es cierto que a determinados enclaves será difícil trasladar los equipos necesarios para este cometido, para lo cual, en todo caso, sería necesario contar con profesionales de la escalada.

El colofón de estas actuaciones sería la posible apertura a la visita de algunos de los yacimientos, al menos aquellos que sus condiciones de accesibilidad no supongan un auténtico riesgo y donde los restos conservados sean visibles y atractivos para el público no especializado. Buenas circunstancias de accesibilidad ofrecen los abrigos del Cerro de la Llana, en un emplazamiento espectacular y con un acceso relativamente fácil, y algunos emplazamientos del valle del Quiebrajano, donde el paseo representa ya en sí mismo una experiencia muy gratificante, y es un lugar habitual de visita para giennenses y foráneos.

\section{BIBLIOGRAFÍA}

CARRASCO RUS, J. et al. (1980): "Las pinturas rupestres del "Cerro de la Pandera" (Jaén). Aproximación al fenómeno esquemático subbético giennense", Publicaciones del Museo de Jaén 5 . Jaén.

et al. (1981): "Avance al estudio de las pinturas rupestres esquemáticas de la Cueva del Plato. Panel A (Otíñar, Jaén)", Zephyrus XXXII-XXXIII.

— et al. (1981): "Las pinturas rupestres esquemáticas del abrigo de La Higuera (Otíñar, Jaén)", Archivo de Prehistoria Levantina XVI.

_ (1982): "Panorama arqueológico de la provincia de Jaén", Publicaciones del Museo de Jaén, Jaén. (1983): "Excavaciones en el complejo cavernícola de El Canjorro, Jaén”, XVI C.N.A., Zaragoza.

- et al. (1985): El fenómeno rupestre esquemático en la cuenca alta del Guadalquivir, I: las sierras subbéticas, Prehistoria Giennense, 1 Jaén.

CHICOTE UTIEL, M. et al. (1973): “Nuevas pinturas rupestres en Jaén”, Boletín del Instituto de Estudios Giennenses LXXVIII. Jaén.

CHOCLÁN SABINA, C. (1988): Catálogo de yacimientos arqueológicos de la provincia de Jaén, Consejería de Cultura, inédito.

HORNOS MATA, F. (1992):"Memoria de gestión de las actividades arqueológicas de la provincia de Jaén", A.A.A. '90, I, Sevilla.

ISTITUTO CENTRALE PER IL RESTAURO (1992): La Carta de Riesgo, Consejería de Cultura y Medio Ambiente. Junta de Andalucía, Cádiz, 1992.

LÓPEZ PAYER, M. G. et al. (1993): "Reproducción y estudio directo del arte rupestre en los términos de Jaén y Quesada (Jaén)", A.A.A. '90, II Actividades Sistemáticas, Sevilla.

- et al. (1994): "Historia de la investigación del arte rupestre prehistórico en la provincia de Jaén, Alto Guadalquivir: trabajos de campo y metodología científica", Boletín del Instituto de Estudios Giennenses CLIII, Jaén.

LÓPEZ RUIZ, E. (1972): “Gran descubrimiento", Cimas. La revista de los deportes de montaña, Año I, n 5. MÁS CORNELLÁ, M.: Catálogo de yacimientos con pinturas rupestres en Andalucía, Consejería de Cultura, 1994, inédito. 
RODRÍGUEZ DE GUZMÁN SÁNCHEZ, et al. (2002a): "La tutela del Patrimonio Histórico a través de las Cartas Arqueológicas municipales", Boletín del Instituto Andaluz del Patrimonio Histórico, en prensa, Consejería de Cultura, Junta de Andalucía.

(2002b): "La Gestión del Arte Rupestre en Andalucía. Actuaciones en materia de Protección y Conservación", Revista Panel 1. Consejo de Arte Rupestre del Arco Mediterráneo. Junta de Andalucía. Consejería de Cultura (coord.). Sevilla.

SÁNCHEZ MARTÍNEZ, C. et al. (1983): "Las pinturas rupestres de "La Cantera" en Otíñar (Jaén)”, XV C.N.A., Zaragoza.

SANTANA FALCÓN, I. (2001): "Evaluación de factores de riesgo en las estaciones con arte rupestre de la provincia de Málaga", Boletín del Instituto Andaluz del Patrimonio Histórico 36, Consejería de Cultura, Junta de Andalucía.

SERRANO PEÑA, J. L.: Inventario de yacimientos arqueológicos de la provincia de Jaén, Consejería de Cultura, 1993, inédito.

SORIA LERMA, M. (1989): El arte rupestre en el sureste de la Península Ibérica, La Carolina.

- (1991): "El arte rupestre primitivo al Sur de Jaén", Entorno a la cultura, la información y el deporte, Revista de la Obra Socio-Cultural de la Caja de Jaén, Jaén.

ZAFRA DELA TORRE, N. (1999): "El patrimonio arqueológico como recurso socio económico: El proyecto Otíñar", Revista Sumuntan 11.

(2000): "Dolmen y pinturas rupestres de Otínar(Puente de la Sierra)", Visitas al Patrimonio Histórico Provincial de Jaén. Ed. Colegio Oficial de Arquitectos de Jaén. 